\title{
Intubating laryngeal mask airway as an independent ventilatory and intubation device. A comparison between supine, right lateral and left lateral
}

\author{
Mamta Panwar ${ }^{1}$, Avnish Bharadwaj ${ }^{2}$, Gaurav Chauhan ${ }^{1}$, and Drubajyoti Kalita ${ }^{1}$ \\ Department of Anesthesiology, ${ }^{1}$ VMMC \& Safdarjang Hospital, New Delhi, ${ }^{2}$ SMS Medical College, Jaipur, India
}

Background: Sudden loss of airway in patients in the lateral position has always been proven to be difficult to manage with conventional laryngoscopy. We performed a randomized controlled trial to prove the success rate of ventilation and intubation in the lateral position via intubating laryngeal mask airway (ILMA).

Methods: Ninety patients were divided into three groups of 30 patients each, positioned supine, right lateral, and left lateral randomly. Each group comprised of both sexes of American Society of Anesthesiologists grade I and II, aged between 18-55 years with normal airway posted for surgery under general anesthesia. Patients were pre-medicated with fentanyl followed by induction with propofol and neuromuscular blockade with rocuronium. ILMA was inserted and blind tracheal intubation via ILMA was done. The success rate, time taken and the number of adjusting maneuvers used for both procedures were recorded. The data was tabulated and analyzed using ANOVA (analysis of variance), multiple ' $t$ ' test and chi square.

Results: The success rate of intubation (96\%) and time taken in insertion and intubation was found to be quite similar in all the three groups.

Conclusions: We conclude that the ILMA has an important role to play in the emergency management of airways in patients in the lateral position in terms of ease, success rate and time taken. (Korean J Anesthesiol 2013; 65: 306-311)

Key Words: Endotracheal intubation, Fentanyl, Propofol.

Received: August 17, 2012. Revised: 1st, September 29, 2012; 2nd, November 1, 2012. Accepted: November 6, 2012.

Corresponding author: Mamta Panwar, M.D., Department of Anesthesiology, VMMC \& Safdarjang Hospital, A-6, Type IV, SJH Staff Quaters, West Kidwai Nagar, New Delhi 110023, India. Tel: 91-9013000543, Fax: 91-11-26163072, E-mail: drkumath@gmail.com

(a) This is an open-access article distributed under the terms of the Creative Commons Attribution Non-Commercial License (http:// creativecommons.org/licenses/by-nc/3.0/), which permits unrestricted non-commercial use, distribution, and reproduction in any medium, provided the original work is properly cited. 


\section{Introduction}

Airway management often acquires precedence over other treatment modalities in any emergency situation.

The technique of endotracheal intubation has become the gold standard for airway management of patient who are unconscious. Intubation of the trachea not only provides an opportunity for controlled ventilation even for prolonged periods in any position, but also offers the opportunity for removal of tracheal secretions.

Regularly, there are case reports regarding accidental extubation during general anesthesia, loss of airway under regional anesthesia, and also where block patients required general anesthesia, due to inadequate effect of block while they were laterally placed [1-3]. In these cases where patients were placed laterally, conventional laryngoscopy failed to intubate the trachea or took a longer time [2,3] but light guided tracheal intubation via intubating laryngeal mask airway (ILMA) has been performed successfully [3].

A comparative study on the feasibility of ILMA placement and blind intubation via the ILMA between patients placed in the supine and the lateral position has been performed $[4,5]$.

We did a randomized prospective study to compare the feasibility of ILMA as an independent ventilatory device and conduit for blind tracheal intubation in patients placed in the supine and lateral position (right and left both) with respect to success rate and time taken.

\section{Materials and Methods}

With the permission from competent authorities (Medical Superintendent of SMS MC, Jaipur) during 2003-2007, year with written consent of patients, 90 adult patients scheduled for general surgery under general anesthesia (American Society of Anesthesiologists [ASA] grade I and II) of both sexes aged between 18-55 years were randomly enrolled using computer generated randomized numbers.

Exclusion criteria for study were history of cardio -respiratory disease (e.g. hypertension, chronic obstructive pulmonary disease, ischemic heart disease), cerebro-vascular disease, reflux oesophagitis, symptomatic hiatus hernia, peptic ulceration or non- fasting.

Also excluded were patients with predicted difficult airways or inadequate mouth opening.

Along with other parameters, Mallampati scoring, mouth opening, Thyromental distance and Sternomental distance with head extension in the upright and sitting position were recorded.

Peri-operative monitoring included electrocardiogram, heart rate, non invasive blood pressure, capnography and pulse oximetry.
Before induction of anesthesia, patients were randomly divided into 3 groups. Each group comprised of 30 patients were positioned right lateral, left lateral and supine.

The feasibility of ventilation was assessed by means of bag and mask ventilation before placing them right or left lateral under supervision of a senior anesthesiologist. The head was supported on a pad $8 \mathrm{~cm}$ thick (with added padding, if needed) in the neutral position and oxygen was administered via a facemask for 3 minutes before giving anesthesia.

Patients were pre-medicated with glycopyrolate $0.2 \mathrm{mg}$ intraveneous (IV) and fentanyl in a dose of $1 \mu \mathrm{g} / \mathrm{kg}$ IV and pre oxygenated using $100 \% \mathrm{O}_{2}$. Patients were induced with intravenous propofol $3 \mathrm{mg} / \mathrm{kg}$ IV injected over 30 seconds and maintained with isoflurane in oxygen. ILMA was inserted when eyelash reflex was lost and the jaw was relaxed. Additional bolus of propofol 10-20 mg IV was allowed until an adequate level of anesthesia was achieved.

Standing above patients head, ILMA was inserted into the hypo-pharynx, with the insertion technique consisting of a one handed rotational movement in the saggital plane, with head supported by a pillow to achieve a neutral position as described by Brain et al. [6]. Size 4 ILMA was used for both sexes; after insertion, the cuff was inflated with $30 \mathrm{ml}$ of air. Successful placement was confirmed by positive capnography along with ease of ventilation and adequate chest wall expansion.

The time required to insert the ILMA was measured from the time of introduction of ILMA between the incisors, till the successful restoration of ventilation.

If ventilation through the ILMA produced resistance, leakage and/or unsatisfactory ventilation, optimization of ventilation was perfomed using adjusting maneuvers in the following order (I) Chandy's maneuver-consisting of rotating the device in the saggital plane followed by slightly lifting the handle of the ILMA away from the posterior pharyngeal wall (II) extension maneuver-pulling the handle back towards the intubator (III) flexion maneuver - pushing the handle of ILMA away from the intubator.

In case of a failed insertion, three attempts were allowed. Meanwhile, in between attempts, patients were oxygenated by face mask. After 3 attempts, as well as in the case of desaturation $\left(\mathrm{SpO}_{2}<92 \%\right)$ with hemodynamic instability, patients were moved to the supine position and intubated by conventional laryngoscopy. The Cormack \& Lehane laryngoscopy score was noted in these cases.

The number of attempts for successful placement of ILMA, number/type of adjusting maneuvers used, and time needed for final attempt to the ILMA insertion were noted.

After confirming satisfactory ventilation with positive capnography rrocuronium $0.6 \mathrm{mg} / \mathrm{kg} \mathrm{IV}$ was administered. Once adequate muscle relaxation (after $120 \mathrm{sec}$ ) is achieved, lubricated 
Table 1. Demographic and Airway Assessment Data

\begin{tabular}{lccc}
\hline \multicolumn{1}{c}{ Parameters } & Supine group $(\mathrm{n}=30)$ & Rt. Lat. group $(\mathrm{n}=30)$ & Lt. Lat. group $(\mathrm{n}=30)$ \\
\hline Weight $(\mathrm{kg})$ & $57.52(9.08)$ & $56.27(9.49)$ & $51.68(11.99)$ \\
Height $(\mathrm{cm})$ & $160.31(10.42)$ & $156.04(9.27)$ & $154.51(9.83)$ \\
Sex $(\mathrm{M} / \mathrm{F})$ & $10 / 20$ & $9 / 21$ & $7 / 23$ \\
Mallampati I/II/III/IV & $30 / 0 / 0 / 0$ & $27 / 3 / 0 / 0$ & $29 / 1 / 0 / 0$ \\
Thyromental distance $(\mathrm{cm})$ & $6.91(0.80)$ & $7.06(1.78)$ & $6.82(1.06)$ \\
Sternomental distance $(\mathrm{cm})$ & $17.66(1.55)$ & $17.95(1.71)$ & $18.40(1.79)$ \\
Inter-dental distance $(\mathrm{cm})$ & $4.00(0.40)$ & $3.97(0.76)$ & $4.23(0.94)$ \\
\hline
\end{tabular}

Data presented as mean (SD) or number of patients.

Table 2. Intubating Laryngeal Mask Airway Insertion and Intubation Data

\begin{tabular}{|c|c|c|c|c|}
\hline Parameters & Supine group $(\mathrm{n}=30)$ & Rt. Lat. group $(\mathrm{n}=30)$ & Lt. Lat. group $(\mathrm{n}=29)$ & $P$ value \\
\hline ILMA insertion time (s) & $27.30(15.86)$ & $26.27(12.47)$ & $24.10(10.33)$ & NS \\
\hline ILMA insertion attempts (n) & & & & NS \\
\hline 1 & 28 & 30 & 24 & \\
\hline 2 & 2 & 0 & 4 & \\
\hline 3 & 0 & 0 & 1 & \\
\hline Successful ILMA insertion (n) & $30(100 \%)$ & $30(100 \%)$ & $29(96.6 \%)$ & NS \\
\hline Intubation attempts (n) & & & & NS \\
\hline 1 & 28 & 25 & 27 & \\
\hline 2 & 1 & 3 & 2 & \\
\hline 3 & 0 & 1 & 0 & \\
\hline Overall intubation success & $29(96.6 \%)$ & $29(96.6 \%)$ & $29(100 \%)$ & NS \\
\hline Time taken in removal of ILMA & $21.47(5.16)$ & $22.63(4.54)$ & $20.62(4.32)$ & NS \\
\hline
\end{tabular}

Data presented as mean (SD) or number of patients. NS: not significant.

silicone tracheal tube size 7.5 was advanced relying on tactile sensation through ILMA. If no obstruction was observed, the tube was inserted $7 \mathrm{~cm}$ beyond the transverse mark. In case of resistance adjusting up-down maneuvers, extension \& optimization were used.

The intubation time (time taken for insertion of the tip of tracheal tube into the metallic end of ILMA till the restoration of ventilation) along with number of attempts, number of adjusting maneuvers used, and success rate were recorded.

Data on patient demographics, number of attempts, number of adjusting maneuvers, success rate and vital signs were recorded, tabulated and analyzed using analysis of variance (ANOVA) and multiple $t$ test for descriptive data between the two groups and chi square for quantitative data considered $\mathrm{P}<0.05$ was statistically significant. Statistical analysis was performed using statistical software SPSS 11.0 version.

\section{Results}

The Table 1 concludes demographic and airway assessment data. We included 30 patients for each group. The ILMA placement was successful in all but one patient (89/90) as shown in Table 2. We performed ILMA insertion in the 1st attempt in $82 / 90$, while 6 required a 2 nd attempt and one occurred at the 3 rd attempt. There was $100 \%$ success rate in the supine and right
Table 3. Time Taken in Intubation through ILMA

\begin{tabular}{lc}
\hline Time & No of cases \\
\hline$<10$ & 8 \\
$11-20$ & 62 \\
$>30$ & 17 \\
Total & 87
\end{tabular}

Data presented in terms of number of patients.

lateral group for ILMA insertion. Only in one patient of the left lateral group we were not satisfied with ventilation even after a 3rd attempt, so we labeled it as a failure.

Time taken for ILMA insertion was quite comparable in all three groups i.e. $27.30 \mathrm{sec}$ in the supine group, 26.27 seconds in the right lateral group and 24.10 in the left lateral group.

As shown in Table 2, intubation via the ILMA was possible in $29 / 30(96.7 \%)$ in both the supine and right lateral group, while $29 / 29(100.0 \%)$ patients were successfully intubated in the left lateral group. Out of these, $28 / 29(96.6 \%)$ in the supine group were intubated in the 1st attempt; $25 / 29$ (86.2\%) in the right lateral group and $27 / 29(93.1 \%)$ in the left lateral group. In the majority of patients $62 / 87$, successful intubation was achieved in 11-20 sec (Table 3). The Intubation time in the supine group was 16.45 (15.17) sec, in the right lateral group was 21.96 (15.27) sec; and in the left lateral group was 20.48 (17.67) sec. There was 
no significant difference between the three groups.

Despite all adjustments, intubation could not be performed successfully in one patient in both the supine and right lateral group.

Time taken in removal of ILMA was 21.47 (5.16) sec in the supine group; 22.63 (4.54) sec in the right lateral group; 20.62 (4.32) sec in the left lateral group.

In only one instance we observed persistent esophageal intubation, which was also true in the supine group. This was a case where in addition to placing ILMA successfully in the second attempt, the ventilation was not satisfactory without manipulations.

We observed one case of accidental extubation whish was also due to slippage of the over-lubricated tracheal tube. Three of our patients complained of a sore throat in the post operative period, in which case we took more than one attempt in doing blind intubation and few adjusting maneuvers were used as shown as number of attempts in Table 2. The ILMA insertion, the blind tracheal intubation through it, and its removal results in increase of heart rate. Though these changes were statistically significant, they were transient and were tolerated fairly well with patients of ASA status I and II (Fig. 1).

The ILMA insertion and tracheal intubation results in a transient but significant increase in systolic blood pressure tolerated uneventfully by patients not having any systemic cardiac disease (Fig. 2).

Oxygen saturation does not decrease below $95 \%$ at any time during the study. No significant trauma to soft tissue was recorded.

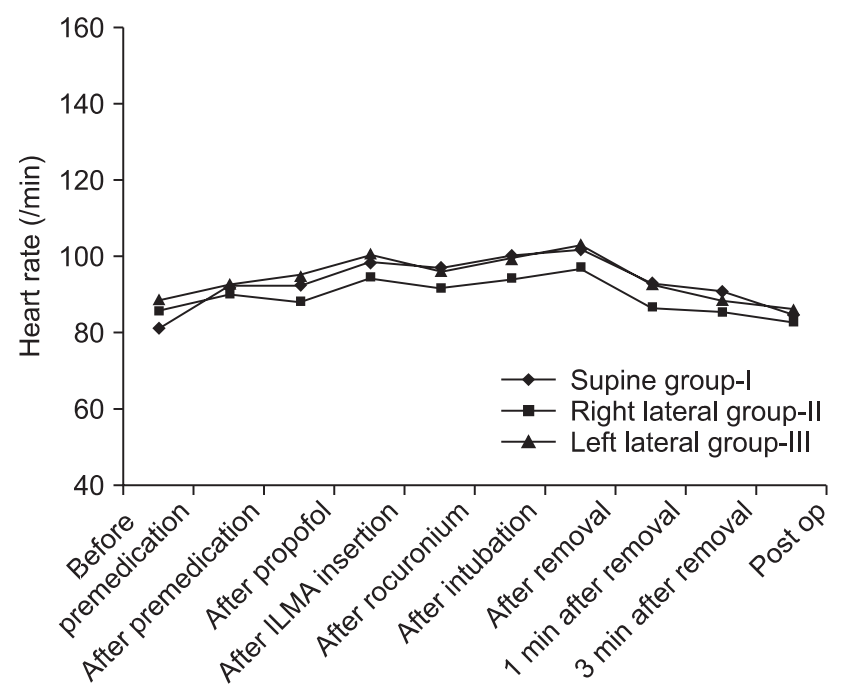

Fig. 1. Changes in heart rate in different groups.

\section{Discussion}

This randomized prospective controlled study was designed to evaluate the feasibility of the use of ILMA in a simulated emergency situation of sudden accidental loss of airway during the middle of surgery in patients lying in the lateral position. Such a scenario can be extrapolated to disaster conditions where the victims may be trapped in a lateral position under the rubble following an earthquake. Conventional laryngoscopy has limitations in right lateral patients due to blade design. Also, lateral positioning results in deterioration of the laryngoscopic view which can be disastrous in case of failed intubation skills [7].

Our study was extrapolated to review the importance of ILMA in laterally placed patients with respect to success rate and intubation time.

We concluded that the success rate of insertion and adequate ventilation through ILMA was almost equal in the supine as well as right and left lateral groups. We successfully inserted ILMA in all but one patient among 89/90 (i.e. 98.99\%) patients, with ease of insertion in 79 and a little bit of adjustment in the remaining 10 patients, besides the gravity-dependent descent of the paralyzed lower jaw in the lateral position. The success rate of ventilation via ILMA in the lateral position was similar to that of standard LMA [8].

The success rate of intubation in the first attempt was higher in the supine group (96.55\%), but comparable with the right lateral (86.20\%) and left lateral groups (93.10\%). Despite all the maneuvers we could not intubate trachea in two patients, one from each group (supine and right lateral). The patient in the supine group whose trachea could not be intubated was the one with unsatisfactory ventilation, and suggests improper place-

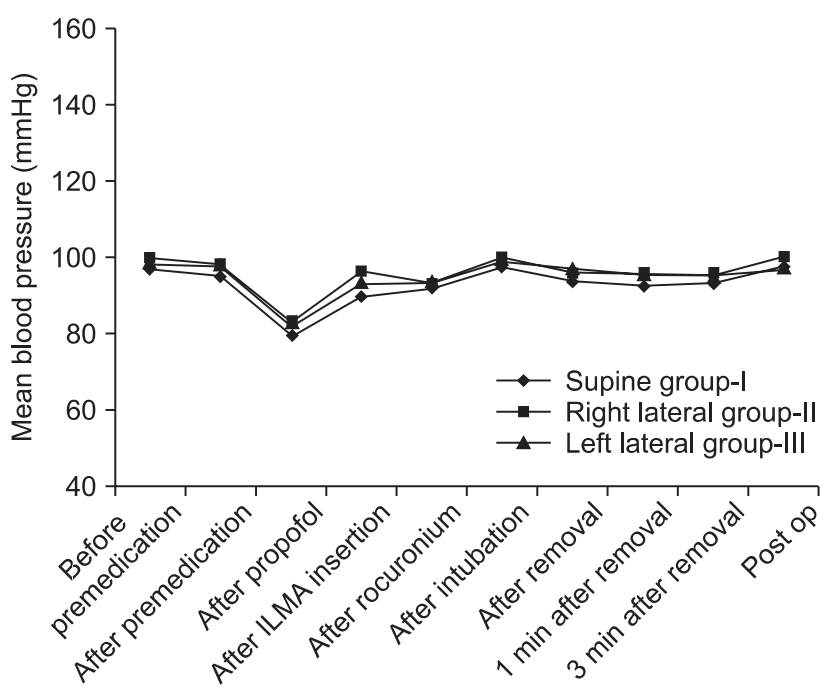

Fig. 2. Changes in mean blood pressure in different groups. 
ment of ILMA leading to failure in achieving intubation through it.

The overall success rate of blind intubation in the lateral position at $96.6 \%$ did not differ from similar studies $[5,9,10]$. Additionally our results for supine patients are quite comparable with light-wand guided intubation via ILMA in supine patients [11-14]. Fibreoptic guidance definitely improves success rates as reported by Joo and Rose [15], where he compared blind versus fibreoptic intubation via ILMA and by Weiss et al. [16] who used fibre-optic guidance in pediatric patients after a failed blind intubation.

However, as we were simulating situations where intubation was required as emergency rescue measures in patients in the lateral position, our technique proves to be blind but technically rapid and easy (Table 3 ).

The majority of patients in all 3 groups were intubated within 11-20 sec which is quite comparable with previous studies $[10,15]$. In patients where few maneuvers were used to perform successful intubation, it also held true that a few more seconds were required for intubation. The time required for intubation was quite similar in all the three groups ( $20 \mathrm{sec})$. Definitely among the 3 groups, we took the least time in the supine group, but this was due to more familiarity with performing procedures in supine patients.

We observed that Chandy's maneuver is useful in increasing the success of proper placement of ILMA as well as for intubation through it.

While removing ILMA over the tracheal tube, accidental extubation occurred in one patient due to slippage of an over- lubricated silicone tracheal tube. Time taken for removal of ILMA in all the three groups was almost equal.

The ILMA's insertion, intubation and removal resulted in increase in heart rate as well as systolic blood pressure for a transient period, which was well tolerated by patients of ASA grade I and II.

The ILMA is fairly suitable as a ventilatory device in the lat- eral position on apneic patients in the hands of inexperienced personnel.

The blind intubation through ILMA is feasible in a high percentage of patients.

Esophageal intubation occurred irrespective of lateral position due to slippage of the over- lubricated tracheal tube at the time of removal of ILMA in one case, comparable to that of $\mathrm{Ca}$ ponas [11] where he observed that esophageal intubation occurs in $5 \%$ of cases $[11,17]$.

As reported by Komatsu et al. [10], we also expected difficulties for a right- handed investigator to insert ILMA in the lateral position. But we observed that the position of the patient did not affect the success rate of ILMA insertion and intubation through it. Instead, we used a little thrust on the lower jaw to facilitate insertion of ILMA.

Among our patients, three had sore throats postoperatively, which were the cases where we took more than one attempt at intubation.

To conclude, the ILMA has proven to be an effective ventilatory device and a suitable conduit for blind tracheal intubation even in patients lying in the lateral position. Irrespective of age \& sex difference we used size 4 ILMA in all cases due to limitation of resources. As per the record, most of our patients weighed between $41-70 \mathrm{~kg}$, showing that size 4 is suitable for emergency rescue airway management in an average adult Indian population.

The ILMA has an important role to play in the emergency management of airways in patients in the lateral position where other conventional methods of airway management may be difficult or have failed.

\section{Acknowledgments}

The authors acknowledge the support and encouragement of Dr. Meenakshi Sharma, Dr. Amit Kulshreshtha and Dr. Manish Kumath for successful completion of the study.

\section{References}

1. Goldik Z, Mecz Y, Bornstein J, Lurie A, Heifetz M. LMA insertion after accidental extubation. Can J Anaesth 1995; 42: 1065.

2. Riley RH, Swan HD. Value of the laryngeal mask airway during thoracotomy. Anesthesiology 1992; 77: 1051.

3. Dimitriou V, Voyagis GS. Use of the intubating laryngeal mask for airway management and light-guided tracheal intubation in the lateral position. Eur J Anaesthesiol 2000; 17: 395-7.

4. Nathanson MH, Gajraj NM, Newson CD. Tracheal intubation in a manikin: comparison of supine and left lateral positions. Br J Anaesth 1994; 73: 690-1.

5. Biswas BK, Agarwal B, Bhattacharya P, Badhani UK, Bhattarai B. Intubating laryngeal mask for airway management in lateral decubitus state: Comparative study of right lateral and left lateral positions. Br J Anaesth 2005; 95: 715-8.

6. Brain AI, Verghese C, Addy EV, Kapila A. The Intubating laryngeal mask. I: Development of a new device for intubation of the trachea. Br J Anaesth 1997; 79: 699-703. 
7. McCaul CL, Harney D, Ryan M, Moran C, Kavanagh BP, Boylan JF. Airway management in the lateral position: a randomized controlled trial. Anesth Analg 2005; 101: 1221-5.

8. Chen CH, Lin CC, Tan PP. Clinical experience of laryngeal mask airway in lateral position during anesthesia. Acta Anaesthesiol Sin 1995; 33: 31-4.

9. Kihara S, Watanabe S, Taguchi N, Suga A, Brimacombe JR. A comparison of blind and light wand-guided tracheal intubation through the intubating laryngeal mask. Anaesthesia 2000; 55: 427-31.

10. Komatsu R, Nagata O, Sessler DI, Ozaki M. The intubating laryngeal mask airway facilitates tracheal intubation in the lateral position. Anesth Analg 2004; 98: 858-61.

11. Caponas G. Intubating laryngeal mask airway. Anaesth Intensive Care 2002; 30: 551-69.

12. Chan PL, Lee TW, Lam KK, Chan WS. Intubation through intubating laryngeal mask with and without a light wand: a randomized comparison. Anaesth Intensive Care 2001; 29: 255-9.

13. Dimitriou V, Voyagis GS. Use of a prototype flexible lighted catheter for guided tracheal intubation through the Intubating laryngeal mask. Anesth Analg 1999; 89: 257-8.

14. Dimitriou V, Voyagis GS. Use of intubating laryngeal mask for airway management and light-guided tracheal intubation in the lateral position. Eur J Anaestheiol 2000; 17: 395-7.

15. Joo HS, Rose DK. The intubating laryngeal mask airway with or without fiberoptic guidance. Anesth Analg 1999; 88: 662-6.

16. Weiss M, Schwarz U, Dillier C, Fischer J, Gerber AC. Use of the intubating laryngeal mask in children: an evaluation using video-endoscopic monitoring. Eur J Anaesthesiol 2001; 18: 739-74.

17. Dimitriou V, Voyagis GS. Blind intubation via the ILMA: what about accidental esophageal intubation? Br J Anaesth 1999; 82: 478-9. 